American J. of Engineering and Applied Sciences 4 (2): 209-213, 2011

ISSN 1941-7020

(C) 2011 Science Publications

\title{
Experimental Study of Electromagnetic Environment in the Vicinity of High Voltage Lines
}

\author{
${ }^{1}$ Wafa Tourab, ${ }^{2}$ Abdesselam Babouri and ${ }^{2}$ Mohamed Nemamcha \\ ${ }^{1}$ Department of Electrical Engineering, University of Annaba, \\ ${ }^{2}$ Department of Electrical Engineering, Laboratory LGEG, \\ University of Guelma, Algeria
}

\begin{abstract}
Problem statement: Several studies have investigated the impact of electromagnetic field generated by high voltage power lines on environment. The evaluation of electric and magnetic field quantities are of paramount interest in the characterization of electromagnetic environment of the proposed line. The purpose of this study was to analyze the behavior of electromagnetic fields generated by power lines in an area of strong population. Approach: The method applied in this study was gradual; examined the effects of different couplings between the different neighboring lines on behavior of electric and magnetic fields. Later, these lines will be used as a source of disturbance applied to the auditory implants EMC. Results: The implications of experimental results were also compared and discussed with new standard. Conclusion: The maximum intensities of electric and magnetic fields measured for the circuit of three lines close to each other, within the El-HADJAR substation were significantly lower than the ICNIRP reference levels for occupational and no occupational exposures.
\end{abstract}

Key words: Electromagnetic compatibility, low frequency, high voltage transmission lines, inductive coupling, capacitive coupling, voltage lines, electrical energy, electromagnetic fields

\section{INTRODUCTION}

Electrical energy can be considered indispensable for all areas of society, industrial, domestic and social, in such a way that it is difficult to imagine a human activity that does not have some relationship with Electricity (E). Electrical energy is transported from the power station to the substations through overhead electric lines and from there to the final users through distribution networks of medium and low voltage. Energy is transported by the electromagnetic field that is propagated throughout the line, since the highintensity electromagnetic fields can induce important voltages and currents in conducting elements located in their proximity. Many publications have studied the effects of electromagnetic fields and human safety in the vicinity of power lines and stations of mobile communication (Chiang and Tam, 2008) and for the evaluation of electromagnetic fields and modeling of electrical coupling (Djalel et al., 2007).The 2007 Eurobarometer survey showed a strong concern and preoccupation of the European population to electromagnetic fields (The Electromagnetic Fields Special Eurobarometer 272a/Vague 66.2-TNS), the effects of electromagnetic fields on human health are discussed and existing scientific researchers agree more on the precautionary principle, which is an approach based on the idea that absence of evidence of harm, precaution must be (Hender and Soltan, 2007). However, the relationship between the electromagnetic fields exposure and occurrence of alleged diseases has not yet been proved. In this context various occupational health organizations have established limits of exposure to EM fields in the study and public places. Among these organizations are cited the ICNIRP and standard IEEE guidelines which set a basic restriction on the density of electric current induced in the body by ELF fields (ICNIRP, 1998) mainly to avoid electrical shock hazards, minimize burn hazards and reduce interference with medicals implants (Babouri and Hedjiedj, 2007). To characterize low frequency electromagnetic fields generated by high voltage lines, some authors have developed mathematical models for the characterization of the electromagnetic environment in the vicinity of power lines, based on solving Maxwell's equations and Laplace for simple geometries. In practical terms, these models are not the most suitable due to their complex mathematical and inaccuracy in the calculations. This justifies the choice of experimental technique with well-defined exposure conditions to determine the levels of electric and magnetic fields near power lines. Experimental 
characterization of low frequency electromagnetic fields has been conducted in several publications (Paraskevopoulos et al., 2009; Nor et al., 2008; Cipo et al., 2005; National Radiation Laboratory, 2005). The mastery of techniques for measuring electromagnetic fields radiated by power lines and their compatibility, at our reference center (LGEG), is a precursor to involvement in experimental research on the impact of EMF on the biological environment generally and on health particularly. In this context we conducted this study in collaboration with multidisciplinary research teams: CHU of Annaba (the University Hospital) and SONELGAZ (Algerian electricity company), whose aim is to develop study protocols relating to the electromagnetic fields compatibility. In order to achieve a regional database on the experimental characterization of low frequency electromagnetic fields in the laboratory, this will be made available to the concerned partners. In this study the experimental measurement of electric and magnetic field have been made in the post-El Hadjar which provides interconnection between the Far East region of Algeria and Tunisia, This position has a particular interest given its location in an urban area. It covers a range of voltage 220,90 and $60 \mathrm{kV}$ which will permit us to highlight the effects of inductive and capacitive coupling on the distribution of the magnetic and electric field generated in the vicinity of the line circuits operating in parallel. Experimental results show that the intensities of the fields identified for the proposed model lines are well below allowable limits set by the International Institute for Protection against Radiation Protection (ICNIRP), the latter will be used for the characterization of high voltage lines, as a source of electromagnetic interference of medical implants including auditory implants.

\section{MATERIELS AND METHODS}

Model description: To characterize the electromagnetic environment of power lines, we conducted our investigation in coordination with the Algerian company of electricity (SONELGAZ) at elHadjar electrical post located in eastern Algeria. Measurements have also been carried out under 220 and 90KV lines located next to each other Fig. 1.

The lines B (90KV) and C (220KV) represents respectively SOUK-AHRAS city and EL-KALA city with a flat configuration, in the middle the line A (90KV) represents Mejaz el-Safa city with a vertical configuration. The distance between phase lines, the clearance of conductors, cables Guard, the lengths of lines and the currents in conductors when measuring the electromagnetic field are mentioned in (Table 1). The Rapprochement between the geometric heights of the conductors of lines B $(90 \mathrm{KV})$ and $\mathrm{C}(220 \mathrm{KV})$ is due to it being an old line of $220 \mathrm{kV}$ that is now used at $90 \mathrm{kV}$.

Experimental investigation: Measurements of electric and magnetic fields have been conducted in the free space under the high voltage lines in order to achieve a characterization of the electromagnetic environment and to draw the profile of the electric field and magnetic field near the 220 and $90 \mathrm{kV}$ lines located a few meters from each other, taking into account the inductive and capacitive coupling between the different phases. In literature much mathematical and experimental approaches to obtain the earth resistance values at field site were published (Nor et al., 2008).In our case the earth is considered as a perfect conductor. Then, measurements were made at two meters intervals along a direction perpendicular to the power lines, in starting from the central pylon $\mathrm{A}$ and when moving in an east direction to the centre of pylon $\mathrm{C}$, then in an west direction to the centre of pylon $\mathrm{B}$ using an referenced and calibrated electromagnetic field meter PMM8053A Fig. 2. To avoid the perturbation of electrical field the device is equipped with an isotropic probe mounted on one-meter-high non-conducting tripod.

\begin{tabular}{|c|c|c|c|}
\hline & $\begin{array}{r}\text { Pylon A } \\
(90 \mathrm{kV})\end{array}$ & $\begin{array}{c}\text { Pylon B } \\
(90 \mathrm{kV})\end{array}$ & $\begin{array}{c}\text { Pylon C } \\
(90 \mathrm{kV})\end{array}$ \\
\hline Directions & Mejaz el-Safa & El-Kala & Souk-Ahras \\
\hline Height of the phase $1(\mathrm{~m})$ & 30.60 & 28.20 & 30.8 \\
\hline Height of the phase $2(\mathrm{~m})$ & 33.85 & 28.20 & 30.8 \\
\hline Height of the phase $3(\mathrm{~m})$ & 37.10 & 28.20 & 30.8 \\
\hline Height of the cable guard (m) & 43.60 & 32.80 & 33.3 \\
\hline Phase Spacing $(\mathrm{m})$ & 3.25 & 07.00 & 7.7 \\
\hline Length $(\mathrm{km})$ & 45.70 & 66.75 & 86.4 \\
\hline Nub of conductors by phase & 01.00 & 01.00 & 01.0 \\
\hline Value of current by phase (A) & O(unloaded line) & 84.00 & 70.0 \\
\hline
\end{tabular}

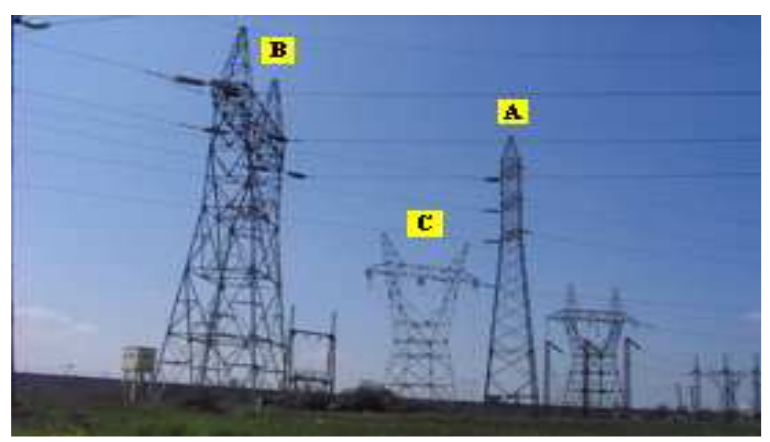

Fig. 1: Model studied 


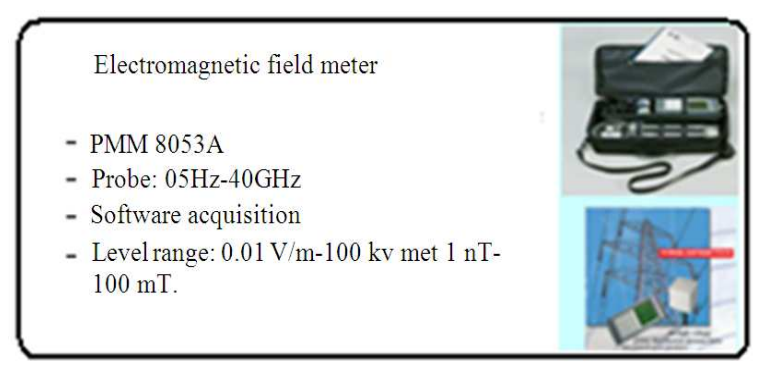

Fig. 2: Characteristics of the measuring equipment

This instrument measures the electric and magnetic fields in three orthogonal directions in the frequency range $5-40 \mathrm{GHz}$, the field strength measured must remain constant. This is the only way to ensure that dangerous field strengths are not present. The acquisition of data was done in real time by the computer software package. The measurement results have been reported in $\mathrm{V} / \mathrm{m}$ for the electric field and $\mu \mathrm{T}$ for the magnetic field in the international system of units.

\section{RESULTS}

The profiles of electric and the magnetic field measurements are shown in Fig. 3-4. They reveal the effects of inductive and capacitive coupling and the changes of the direction of the power lines through different locations on the intensities of fields measured.

Figure 3 clarifies the field strength distribution at $1 \mathrm{~m}$ height above the ground for the circuit of lines considered. This figure reveals that the maximum value of the electric field decrease, when increasing the ground clearance level which is highly dependent on the voltage of the line, while the location at which we measured the maximum values is the centerline of the pylon $\mathrm{C}(220 \mathrm{kV})$. When we move from the right of the line A $(90 \mathrm{kV})$ uncharged, with a vertical arrangement R-S-T of phases to the $7 \mathrm{~m}$ point, the intensity of the electric field generated increases and reaches a maximum value of $401 \mathrm{~V} \mathrm{~m}^{-1}$. In the $14 \mathrm{~m}$ point, $\mathrm{R}$ S-T horizontal arrangement of the phases of the line $B$ causes a cancellation of the quantities of electric field in the intermediate zone between the two lines $\mathrm{A}$ and $\mathrm{B}$, the electric field intensity measured at this point is $166 \mathrm{~V} \mathrm{~m}^{-1}$.

Figure 4 shows the transverse profile of the corresponding magnetic field depending on the distance to the axes of the pylons for the circuit lines $\mathrm{A}, \mathrm{B}$ and $\mathrm{C}$ neighbors, both with the conductors in vertical and horizontal simple arrangement R-S-T.

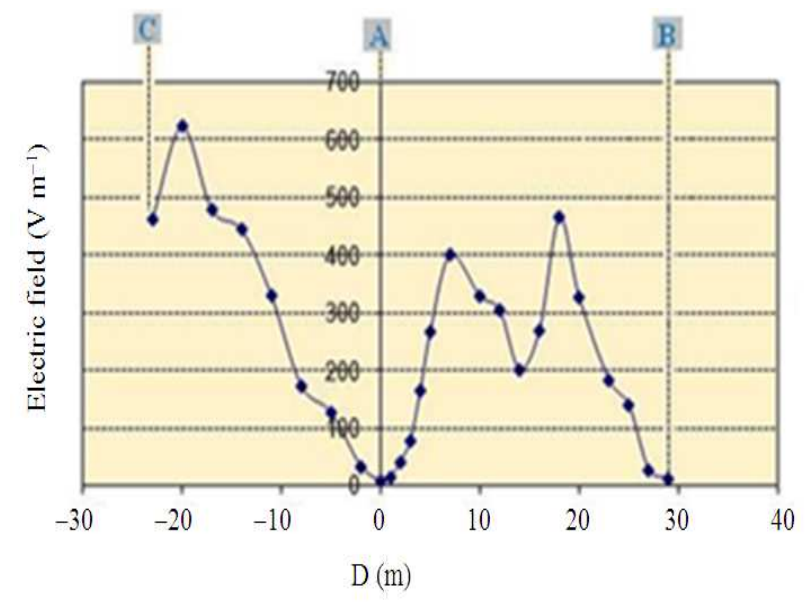

Fig. 3: The profile of electric field

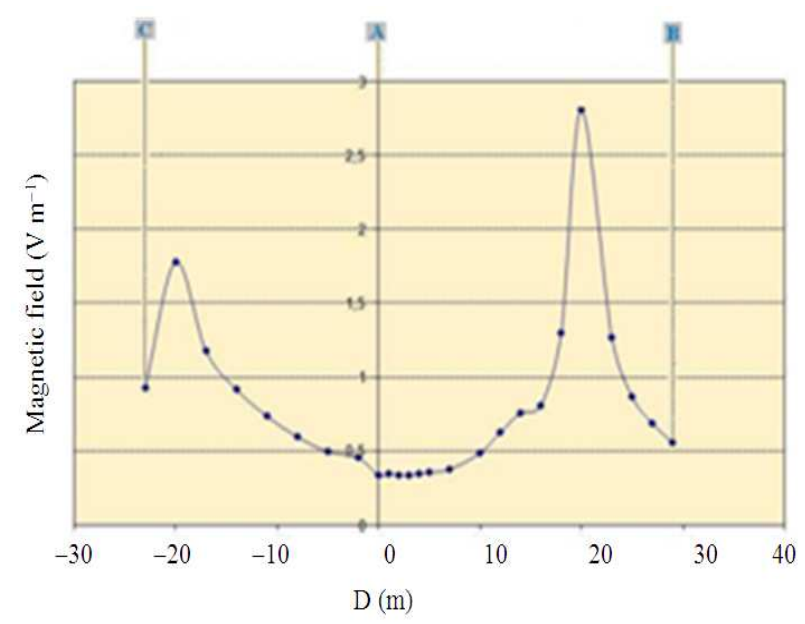

Fig. 4: The profile of magnetic field

The conductors of the line A are located at a height of $30,6 \mathrm{~m}$ with a separation between phases of $3,25 \mathrm{~m}$ and no current flowing through. At the moment of measuring the magnetic field, mean currents of 84-70 A circulated respectively through the conductors of the lines B and C. As is observed in the figure maximum values of the magnetic field are obtained in the center of each line (the centers of the lines are located at $20 \mathrm{~m}$ for the line $\mathrm{B}$ on the right and $-23 \mathrm{~m}$ for the line $\mathrm{C}$ on the left. the maximum value of the field is reached in the center of the line B and is below $(2,81 \mu \mathrm{T}$, this values remain well below the maximum limit $100 \mu \mathrm{T}$ set by European regulations). As can be seen, the value measured under the line $\mathrm{C}$ on the left $(1.78 \mu \mathrm{T})$ is much lower than the previous value. 


\section{DISCUSSION}

To argue ours experimental results, we are based on the tow fundamental principles related to electromagnetic compatibility of high voltage power lines. The effect of electric field to induce currents in the wires parallel to the electric field lines (capacitive coupling) and the effect of magnetic field to induce a voltage in the loops perpendicular to the field lines (inductive coupling). These phenomena could be respectively modulated in an equivalent electrical circuit by mutual capacitances and inductances. It can be seen in the previously figures that the low frequency electromagnetic fields are located close to the line conductors; they have brought space relatively low and field strengths inversely proportional to the distance from the source. The maximum electric field measured is highly depending on the voltages of the lines, further away from the lines the electric field strength decreases rapidly. The intensity of magnetic field measured under the line $\mathrm{C}$ on the left is much lower than this measured under the line $\mathrm{B}$ on the right, evidently, this is due to the fact that a smaller current circulates through the latter and the height at which the conductors of the right line are placed is inferior to that of the conductors of the left line. Power lines located near to each other are coupled; the coupling appears in the profiles of the magnetic and electric field drawn. Lateral distribution of the electric and magnetic field strengths depends on the phase arrangement in power lines (Milutinov et al., 2008) the same phase arrangement R-S-T; R-S-T produces the lowest electric field strength and magnetic flux density in the intermediate zone between both lines than if another arrangement is used.

\section{CONCLUSION}

This experimental investigation has allowed us to make a collection of experimental data on the characterization of electromagnetic fields generated by power lines at low frequencies, to create a database that can be exploited in the transport of electrical energy in Algeria according to international standards. The profiles of electric and magnetic field surveys show the effects of inductive and capacitive coupling on the distribution of quantities of electric and magnetic charges generated by circuits of neighboring lines. The experimental results show that the maximum intensities of these fields have not exceeded the permissible limits set by ICNIRP which correspond to the exposure of the general public has limits of $100 \mu \mathrm{T}$ for the magnetic field (B) and $5 \mathrm{kVm}^{-1}$ for Electric field (E). These results will be used to study the interference of low frequency electromagnetic fields with medical implants (hearing aids), as part of a research project of the laboratory LGEG in collaboration with the CHU (University Hospital) of Annaba.

\section{ACKNOWLEDGMENT}

This study was supported by SONELAZ "Algerian National Company of Electricity". We thank the director of the company Mr. Benkirat Messoud; The engineers design office of the national electricity company of Annaba and team operating of the substation El-Hadjar for their contribution in achieving of this study. We thank Mr. Dafri Mourad LGEG Engineer Laboratory for her technical assistance.

\section{REFERENCES}

Babouri, A. and A. Hedjiedj, 2007. In vitro investigation of eddy current effect on pacemaker operation generated by low frequency magnetic field. Proceedings of the 29th Annual International Conference of the IEEE Engineering in Medicine and Biology Society, Aug. 22-26, IEEE Xplore Press, Lyon, pp: 5683-5686. DOI: 10.1109/IEMBS.2007.4353636

Chiang, K.H. and K.W. Tam, 2008. Electromagnetic assessment on human safety of mobile communication base stations at university of macau. Am. J. Applied Sci., 5: 1344-1347. DOI: 10.3844/ajassp.2008.1344.1347

Cipo, P., M. Braneshi, L. Ahma and E. Hamiti, 2005. Calculation and measurement of the magnetic field of power transmission lines. Polytechnic University of Tirana. http://www.cigrecired.si/Images/File/MIRO/VELENJE2005/C3/CI GREC3-1.pdf

Djalel, D., H. Ali and C. Faycal, 2007. The returnstroke of lightning current, source of electromagnetic fields (Study, analysis and modelling). Am. J. Applied Sci., 4: 42-48. DOI: DOI: 10.3844/ajassp.2007.42.48

Hender, C. and S. Soltan, 2007. Aspects on the influences of the high voltage power lines on the environment. Chem. Bull. POLITEHNICA Univ., 52: $\quad$ 173-176. http://www.chem.utt.ro/buletin_chimie/numere/200 7/art_43\%28173-176\%29.pdf 
Milutinov, M., A. Juhas and M. Prsa, 2008. Electric and magnetic fields in vicinity of overhead multi-line power system. Proceedings of the 2nd International Conference on Modern Power Systems, Nov. 1214, Cluj-Napoca, Romania, pp: 313-316. http://www.stsb.ro/arhiva/MPS2008/index_files/top ic/V\%2012\%20MILUTINOV\%20M.\%20$\% 2020 . p d f$

National Radiation Laboratory, 2005. Measurement of extremely low frequency electric and magnetic fields beneath $66 \mathrm{KV}$ power lines at burnside Park, Christchurch. Ministery of Health, Manatu Houora.

http://www.nrl.moh.govt.nz/faq/burnsidepark.pdf
Nor, N.M., R. Rajab and K. Ramar, 2008. Validation of the calculation and measurement techniques of earth resistance values. Am. J. Applied Sci., 5: 1313-1317. DOI: 10.3844/ajassp.2008.1313.1317

Paraskevopoulos, A.A.P., P.D. Bourikas and C.G. Karagiannopoulos, 2009. Magnetic induction measurements in high voltage centers of 150/20 $\mathrm{kV}$. Measurement, 42: 1188-1194. DOI: DOI: 10.1016/j.measurement.2009.03.007 